\title{
Improved hemodynamics and cardiopulmonary function in patients with inoperable chronic thromboembolic pulmonary hypertension after balloon pulmonary angioplasty
}

Qi Jin, Qin Luo, Tao Yang, Qixian Zeng, Xue Yu, Lu Yan, Yi Zhang, Qing Zhao, Xiuping Ma, Chenhong An, Changming Xiong, Zhihui Zhao* and Zhihong Liu* ${ }^{*}$

\begin{abstract}
Background: Balloon pulmonary angioplasty (BPA) has been demonstrated to improve cardiac function and exercise capacity in patients with inoperable chronic thromboembolic pulmonary hypertension (CTEPH), but its instant impact on cardiopulmonary function has seldom been evaluated. This study aims to determine the safety and efficacy of BPA and its immediate and lasting effects on cardiopulmonary function among CTEPH patients.

Methods: From May 2018 to January 2019, patients with inoperable CTEPH who underwent BPA sessions were consecutively enrolled. Hemodynamics were measured by right heart catheterization, selective pulmonary angiography and BPA were successively conducted. Hemodynamic variables, WHO functional class (WHO-FC), 6-min walk distance (6MWD) and serum NT-proBNP were evaluated before and after BPA sessions during hospitalization. Pulmonary function testing (PFT) and cardiopulmonary exercise testing (CPET) were performed within 1-3 days pre and post BPA to evaluate the effect of BPA on cardiopulmonary function.

Results: Twenty-five patients with inoperable CTEPH who underwent a total of forty BPA sessions were consecutively enrolled. A total of 183 segmental or subsegmental vessels (4.6 \pm 1.9 vessels per session) in 137 segments (3.4 \pm 1.6 segments per session) were dilated. No procedure-related complications occurred. Instant hemodynamics, WHO-FC, 6MWD and NT-proBNP were all significantly improved after a single BPA session. Significant improvement in cardiopulmonary function was also evident as assessed by PFT indexes (forced vital capacity, forced expiratory volume in the first second, maximal voluntary ventilation) and CPET parameters (peak work rate, peak $\mathrm{VO}_{2}$, oxygen uptake efficiency slope). Further analysis among ten CTEPH patients receiving multiple BPA sessions (2-4 sessions) indicated BPA resulted in lasting improvements in hemodynamics and cardiopulmonary function.
\end{abstract}

Conclusions: BPA, a safe and effective approach, can bring instant improvements after a single session and lasting benefits after multiple sessions to hemodynamics and cardiopulmonary function for patients with inoperable CTEPH.

Keywords: Balloon pulmonary angioplasty, Chronic thromboembolic pulmonary hypertension, Cardiopulmonary function

\footnotetext{
*Correspondence: 1250167892@qq.com; zhihongliufuwai@163.com

Center for Pulmonary Vascular Diseases, Fuwai Hospital, National Center for

Cardiovascular Diseases, Chinese Academy of Medical Sciences and Peking

Union Medical College, 167 Beilishi Road, Xicheng District, Beijing 100037,

China
}

(c) The Author(s). 2019 Open Access This article is distributed under the terms of the Creative Commons Attribution 4.0 International License (http://creativecommons.org/licenses/by/4.0/), which permits unrestricted use, distribution, and reproduction in any medium, provided you give appropriate credit to the original author(s) and the source, provide a link to the Creative Commons license, and indicate if changes were made. The Creative Commons Public Domain Dedication waiver (http://creativecommons.org/publicdomain/zero/1.0/) applies to the data made available in this article, unless otherwise stated. 


\section{Background}

Chronic thromboembolic pulmonary hypertension $(\mathrm{CTEPH})$ is a chronic and progressive disease characterized by fibrous stenosis or obliteration of pulmonary vasculature caused by organized thrombus, leading to progressive increase of pulmonary vascular resistance, right heart failure and terminal death [1-4]. The pathogenesis of CTEPH remains largely unknown, coagulation and fibrinolysis dysfunction, immune and inflammatory disorders, genetic susceptibility, defective angiogenesis and vascular remodeling have been reported to collaboratively contribute to the occurrence and development of CTEPH [5]. Although recent years have witnessed tremendous progress in medical therapies, particularly in pulmonary hypertension $(\mathrm{PH})$-targeted therapies [6], CTEPH is still a serious disease with poor prognosis with 1-, 3-, and 5-year survival rates of $90.2 \%, 78.4$, and $64.5 \%$ respectively amongst patients with not-operated CTEPH [7].

Pulmonary endarterectomy (PEA) is recommended as the method of choice for all eligible patients with CTEPH, yet not all can benefit from this potentially curable procedure due to surgical contraindications and potential complications [4, 8, 9]. Balloon pulmonary angioplasty (BPA) was firstly reported to improve cardiac function and 6-min walk distance (6MWD) in 2001 [10], but it was accompanied with fatal reperfusion pulmonary edema and mechanical ventilation requirement, thus being left on the shelf for more than 10 years. Encouragingly, BPA has once again burst into the limelight since three gratifying reports from Japanese centers in 2012, making it as a promising alternative approach to improve hemodynamics and clinical symptoms [11-13]. Targeted therapy with or without BPA has been proposed in the CTEPH treatment algorithm for patients with non-operable CTEPH [4, 14].

Cardiopulmonary exercise testing (CPET) is a useful and non-invasive tool to objectively and safely evaluate the cardiopulmonary function and exercise capacity [15]. Our previous studies have shown CTEPH is associated with impaired ventilation efficiency and exercise capacity which are independent prognostic predictors in patients with CTEPH [16-19]. Improved exercise capacity assessed by CPET 3 months after BPA has been described [20, 21], nevertheless, the assessment was determined late and might be confounded by rehabilitation training or medical therapies. Since there are no reports regarding BPA in China and its instant impact on cardiopulmonary function among hospitalized patients with inoperable CTEPH, this study aims to firstly evaluate the safety and efficacy of BPA in our center and to assess its early effects on cardiopulmonary function in CTEPH patients.

\section{Methods}

\section{Study patients}

We enrolled twenty-five consecutive patients with CTEPH who underwent BPA procedures in the Center for Pulmonary Vascular Diseases, Fuwai Hospital, Chinese Academy of Medical Sciences and Peking Union Medical College from May 11, 2018 to January 4, 2019. The diagnosis criteria of CTEPH, as we previously described, was established as follows: 1) existing precapillary $\mathrm{PH}$ defined as mean pulmonary arterial pressure (mPAP) $\geq 25 \mathrm{mmHg}$ and pulmonary arterial wedge pressure $(\mathrm{PAWP}) \leq 15 \mathrm{mmHg}$ at rest confirmed by right heart catheterization (RHC); 2) evidence of thromboembolic occlusion of the proximal or distal pulmonary vasculature detected by imaging techniques such as computed tomography, magnetic resonance imaging, ventilation/perfusion scintigraphy or pulmonary angiography; 3 ) the above conditions should be obtained after at least 3 months of effective anticoagulation therapy $[22,23]$. Inclusion criteria for BPA procedures: all CTEPH patients who are not suitable or contraindicated for PEA after operability assessment by our multidisciplinary team including PEA surgeons, $\mathrm{PH}$ experts, BPA interventionists and radiologists; Those with age more than 80 years old, severe liver or kidney dysfunction, iodinated contrast agents allergy, oxygen saturation in peripheral blood $<80 \%$, existence of foreign body such as tumor and thrombus in catheter path, severe bleeding and coagulation disorders, life-threatening hypotension and cardiac arrhythmias, and different types of tumors were excluded. Written informed consent was obtained from all patients, and this study was approved by the Fuwai Hospital Ethics Committee and conformed to the Declaration of Helsinki.

\section{Clinical assessment}

Demographic data (age, sex and body mass index), history of deep vein thrombosis (DVT), PH targeted drugs (endothelin receptor antagonists, phosphodiesterase-5 inhibitors, prostanoids, soluble guanylate cyclase stimulators) were fully assessed after admission among all patients. World Health Organization (WHO) functional class (WHO-FC), N-terminal pro-brain natriuretic peptide (NT-proBNP) levels, oxygen saturation $\left(\mathrm{SaO}_{2}\right)$ measured by arterial blood gas, 6MWD, transthoracic echocardiography (TTE), pulmonary function testing (PFT), chest computed tomography and pulmonary ventilation/perfusion scintigraphy before and after each BPA session within 1-3 days were well documented. General TTE parameters such as left ventricular enddiastolic diameter (LVED), right ventricular end-diastolic diameter (RVED) and tricuspid annular plane systolic excursion (TAPSE) were recorded [24]. RHC was performed through the right femoral vein after local 
anesthesia, hemodynamic parameters included right atrial pressure (RAP), right ventricular pressure, PAP [systolic PAP (sPAP), diastolic PAP (dPAP) and mPAP], PAWP, cardiac output (calculated by indirect Fick's principle) and oxygen saturation (vena cava, right atrium, right ventricle and pulmonary artery). Mixed venous oxygen saturation $\left(\mathrm{SvO}_{2}\right)$, cardiac index $(\mathrm{CI})$ and pulmonary vascular resistance (PVR) were calculated according to standard formulas [20,24].

\section{BPA procedures}

Pulmonary angiography was performed selectively in anterior-posterior and lateral (45 degree) projections after RHC to acquire overall view about the position and degree of filling defect in combination with previous ventilation/perfusion scintigraphy. BPA was routinely performed via the right femoral vein by two interventional cardiologists (Operator: ZHZ; Assistant: TY). A

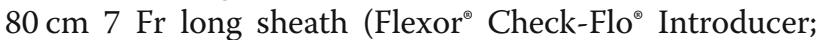
Cook Medical, Bloomington, IN, USA) was inserted into the lobar pulmonary artery to introduce a $6 \mathrm{Fr}$ guiding catheter in order to prevent the guiding catheter from moving along with the beating heart as well as to facilitate the exchange of guiding catheter, wires and balloons. $6 \mathrm{Fr}$ Multi-purpose (Cordis Corporation, Bridgewater, New Jersey, USA), Amplatz Left (mainly for anterior segments, especially A3; Terumo ${ }^{\circ}$ Heartrail $^{\text {TM }}$ II; Terumo Corporation, Tokyo, Japan) or Judkins Right (Terumo $^{\circ}$ Heartrail $^{\mathrm{Ts}}$ II; Terumo Corporation, Tokyo, Japan) was selected as the guiding catheter. After introducing the guiding catheter, $2000 \mathrm{U}$ unfractionated heparin was additionally infused, and oxygen was given at a flow rate of $5-8 \mathrm{~L} / \mathrm{min}$ to all patients. Priority selection of target lesions was as follows: right lung > left lung, inferior lobe $>$ superior or middle lobes, webs or bands > subtotal occlusion $>$ chronic total occlusion $>$ tortuous lesions. A 0.014-in. wire (Hi-Torque Pilot 50; Abbot, Santa Clara, CA, USA) was crossed to the target lesion, then appropriate balloon catheters (Mini Trek; Abbot, Santa Clara, CA, USA) were positioned over the selected lesion and inflated with iodinated contrast agents to pressures of 2-14 atm for 5-30 s. A small-sized balloon $(2.0 \times 20 \mathrm{~mm})$ was firstly used regardless of the diameter of target vessels at initial dilation, smaller balloon may be selected to pass subtotal or total occlusion lesions, inflation pressure was dynamically adjusted according to balloon size and vessel size, and selective angiography with 1:1 mixture of saline and contrast agents was performed with deep breath holds in real time to confirm vascular filling and the presence of ruptured vessels. Dilations were repeated with unchanged or stepwise increased balloon size in case of unresponsive or poorly responsive target angiographic vessel. Hemodynamic parameters were once again measured by $\mathrm{RHC}$ at the end of each BPA session. A typical BPA session lasted for 1$2 \mathrm{~h}$. Each session should not exceed $2000 \mathrm{mGy}$ radiation exposure, $60 \mathrm{~min}$ fluoroscopy time, and $250 \mathrm{ml}$ contrast medium. Complications including reperfusion pulmonary edema, vascular injury and hemoptysis were adjudicated by two interventional cardiologists and were well recorded.

\section{Cardiopulmonary exercise testing}

CPET was performed according to standardized protocol as we previously described $[16,17,25,26]$. An incremental symptom-limited exercise test was performed on an upright cycle ergometer using the COSMED Quark CPET system (COSMED, Rome, Italy). CPET was carried out prior to RHC and after BPA respectively within 3 days, and the protocol used after BPA was identical to that used before RHC. Three minutes of rest was followed by $3 \mathrm{~min}$ of unloaded pedaling and increased exercise using a physician-supervised, progressively increasing work rate (WR) of 5 to $30 \mathrm{~W} / \mathrm{min}$ (the work rate depended on the estimated exercise capacity of each patient) to a maximum tolerance on an electromagnetically braked cycle ergometer. Patients were encouraged to exercise unless they met indications for test termination (eg, inappropriate blood pressure response, severe arrhythmia, dyspnea and leg fatigue). Gas exchange measurements were made by a metabolic cart breath by breath and averaged over $10 \mathrm{~s}$ intervals. Patients were continuously monitored by a standard 12-lead ECG and pulse oximetry $\left(\mathrm{SpO}_{2}\right)$. Blood pressure was recorded using a standard cuff sphygmomanometer every $3 \mathrm{~min}$. Heart rate was measured at 1-min intervals. Peak $\mathrm{VO}_{2}$ was defined as the highest 30 s average of oxygen consumption in the last minute of exercise. Anaerobic threshold (AT) was detected by a combination of the Vslope method and ventilatory equivalents. $\mathrm{PeakVO} / \mathrm{HR}$ was calculated as peak $\mathrm{VO}_{2}$ divided by peak heart rate, and ventilatory efficiency was evaluated by minute ventilation (VE) $/ \mathrm{VCO}_{2}$ slope and $\mathrm{PETCO}{ }_{2} @ \mathrm{AT}$. VE/ $/ \mathrm{VCO}_{2}$ slope was determined by linear regression analysis $(\mathrm{VE}=$ a $\mathrm{VCO}_{2}+\mathrm{b}, \mathrm{a}=$ slope) of $\mathrm{VE} / \mathrm{VCO}_{2}$ plot obtained during exercise. Oxygen uptake efficiency slope (OUES) represented the rate of increase in $\mathrm{VO}_{2}$ in response to a tenfold increase in VE during incremental exercise $\left(\mathrm{VO}_{2}=\mathrm{a}\right.$ $\log 10 \mathrm{VE}+\mathrm{b} ; \mathrm{a}=$ OUES) [25].

\section{Statistical analysis}

All continuous variables were presented as mean \pm SD or median (interquartile range) when appropriate, categorical variables were shown as counts or percentages. A paired $t$ test was used to compare data from the same subject before and after BPA procedures. A two-sided $P$ value $<0.05$ was considered as statistically significant. Paired $t$ test analysis was performed using SPSS version 
23.0 (SPSS Inc., Chicago, IL, USA), and figures displaying changes of variables before and after BPA procedures were drawn using GraphPad Prism version 5.01 (GraphPad Software, San Diego, CA, USA).

\section{Results}

\section{Study population}

A total of 40 BPA sessions were performed in 25 consecutive patients ( 13 male, 12 female, $1.6 \pm 0.9$ sessions per patient) with inoperable CTEPH during the study period. Baseline clinical characteristics of CTEPH patients at the first admission, including hemodynamics measured by RHC before their initial BPA session and PH targeted therapies, were summarized in Table 1. Their mean age at baseline was $58.2 \pm 9.8$ years old, among these CTEPH patients, 8 (32\%) had a past history of DVT. The median time from onset to admission was 3 years. 19 (76\%) patients received $\mathrm{PH}$ targeted medications, $14(56 \%)$ patients received monotherapy and 5 (20\%) patients received combined therapy. Before the first BPA session, 12 (48\%) subjects had symptoms of WHO-FC I/II while 13 (52\%) were in WHO-FC III/IV. The overall CTEPH patients $(n=25)$ had a mean 6MWD of $331.8 \pm 111.3 \mathrm{~m}$ at baseline, with a mPAP of $49.4 \pm 13.4 \mathrm{mmHg}$ and a mean PVR of $952.01 \pm 375.38$ dyn $\cdot \mathrm{s} \cdot \mathrm{cm}^{-5}$.

\section{Efficacy and safety of BPA}

A total of 183 segmental or subsegmental vessels $(4.6 \pm$ 1.9 vessels per session) in 137 segments $(3.4 \pm 1.6$ segments per session) were dilated among 40 BPA sessions. These vessel lesions consisted of 146 webs or bands, 17 subtotal lesions, and 20 total occlusion lesions. After selective pulmonary angiography, a total of 55 balloons $(1.4 \pm 0.5$ per session) matched to the vessel diameters were selected, and balloon diameters and number proportion used for dilation were as follows: $1.2 \mathrm{~mm}(2 \%)$, $1.5 \mathrm{~mm}$ (2\%), $2.0 \mathrm{~mm}$ (47\%), $2.5 \mathrm{~mm}(13 \%), 3 \mathrm{~mm}(18 \%)$, $3.5 \mathrm{~mm}$ (16\%), $4 \mathrm{~mm}$ (2\%). Representative images and videos pre-BPA and post-BPA sessions were displayed in Fig. 1 and Additional file 1. No patients died during the follow-up period till June 1, 2019, and no complications such as pulmonary edema and hemoptysis occurred. The changes in catheter hemodynamics, echocardiographic and clinical parameters before and after BPA sessions $(n=40)$ are shown in Table 2 and Fig. 2. After BPA, CTEPH patients got immediate and significant decreases in sPAP $(84.4 \pm 22.8 \mathrm{mmHg}$ vs $74.7 \pm 21.9$ $\mathrm{mmHg}, P<0.001)$, dPAP $(29.4 \pm 7.8 \mathrm{mmHg}$ vs $25.5 \pm 7.3$ $\mathrm{mmHg}, P<0.001)$ and $\mathrm{mPAP}(47.4 \pm 11.9 \mathrm{mmHg}$ vs $41.5 \pm 10.7 \mathrm{mmHg}, P<0.001)$. However, no significant differences were observed in echocardiographic parameters such as LVED, RVED and TAPSE. Average level of NT-proBNP markedly reduced from $1161.8 \mathrm{pg} / \mathrm{ml}$ to
Table 1 Baseline characteristics of patients with inoperable $\mathrm{CTEPH}$

\begin{tabular}{|c|c|}
\hline Variables & Values \\
\hline Patients, n & 25 \\
\hline Male/Female, n & $13 / 12$ \\
\hline Age, years & $58.2 \pm 9.8$ \\
\hline $\mathrm{BMI}, \mathrm{kg} / \mathrm{m}^{2}$ & $23.6 \pm 3.4$ \\
\hline WHO-FC I/II/III/IV, $\mathrm{n}$ & 1/11/13/0 \\
\hline DVT, n (\%) & $8(32 \%)$ \\
\hline Duration from onset to admission ${ }^{a}$, years & $3.0(1.1-5.5)$ \\
\hline $\mathrm{SaO}_{2}{ }^{a}, \%$ & $91.3 \pm 2.3$ \\
\hline $\mathrm{SvO}_{2}{ }^{\mathrm{a}}, \%$ & $68.9 \pm 5.3$ \\
\hline$m R A P^{a}, m m H g$ & $7.4 \pm 3.3$ \\
\hline mPAPa, mmHg & $49.4 \pm 13.4$ \\
\hline$P V R^{a}$, dyne $\cdot s \cdot \mathrm{cm}^{-5}$ & $952.01 \pm 375.38$ \\
\hline $\mathrm{Cl}^{\mathrm{a}}, \mathrm{l} / \mathrm{min} / \mathrm{m}^{2}$ & $3.18 \pm 0.74$ \\
\hline PAWPa, mmHg & $10.2 \pm 3.0$ \\
\hline PH targeted medication ${ }^{a}, \mathrm{n}(\%)$ & 19 (76\%) \\
\hline Monotherapy, n (\%) & $14(56 \%)$ \\
\hline ERAs & $2(8 \%)$ \\
\hline PDE-5is & $8(32 \%)$ \\
\hline sGCs & $4(16 \%)$ \\
\hline Combined therapy, n (\%) & $5(20 \%)$ \\
\hline ERAs+PDE-5is, n (\%) & $5(20 \%)$ \\
\hline $6 \mathrm{MWD}^{\mathrm{a}}, \mathrm{m}$ & $331.8 \pm 111.3$ \\
\hline Number of BPA sessions, $\mathrm{n}$ & 40 \\
\hline Number of treated segments per session, $n$ & $3.4 \pm 1.6$ \\
\hline Number of treated vessels per session, $n$ & $4.6 \pm 1.9$ \\
\hline Number of balloons per session, $\mathrm{n}$ & $1.4 \pm 0.5$ \\
\hline
\end{tabular}

$B M I$ body mass index, WHO-FC WHO functional class, DVT deep vein thrombosis, $\mathrm{SaO}_{2}$ arterial oxygen saturation, $\mathrm{SvO}_{2}$ mixed venous oxygen saturation, $m R A P$ mean right atrial pressure, $m P A P$ mean pulmonary artery pressure, $P V R$ pulmonary vascular resistance, $\mathrm{Cl}$ cardiac index, PAWP pulmonary arterial wedge pressure, $P H$ pulmonary hypertension, ERAs endothelin receptor antagonists, $P D E-5$ is phosphodiesterase- 5 inhibitors, $s G C s$ soluble guanylate cyclase stimulators, 6MWD 6-min walk distance, BPA balloon pulmonary angioplasty

${ }^{\mathrm{a}}$ Data at the first admission

$671.2 \mathrm{pg} / \mathrm{ml}(P=0.001)$. Moreover, 6MWD dramatically increased from $350.0 \pm 103.4 \mathrm{~m}$ to $403.6 \pm 81.3 \mathrm{~m}$ after BPA treatment. Compared with baseline, WHO-FC improved with the number of patients in I, II, III, and IV changing from $1 / 26 / 13 / 0$ to $8 / 30 / 2 / 0$, respectively.

\section{Cardiopulmonary function after a single BPA session}

As for PFT, forced vital capacity (FVC), FVC\% predicted, forced expiratory volume in the first second (FEV1), FEV1\% predicted, maximal voluntary ventilation (MVV) and MVV\% predicted all significantly improved postBPA compared to those of pre-BPA (all $P<0.05$; Fig. 2). As shown in Table 3 and Fig. 3, exercise performance as 

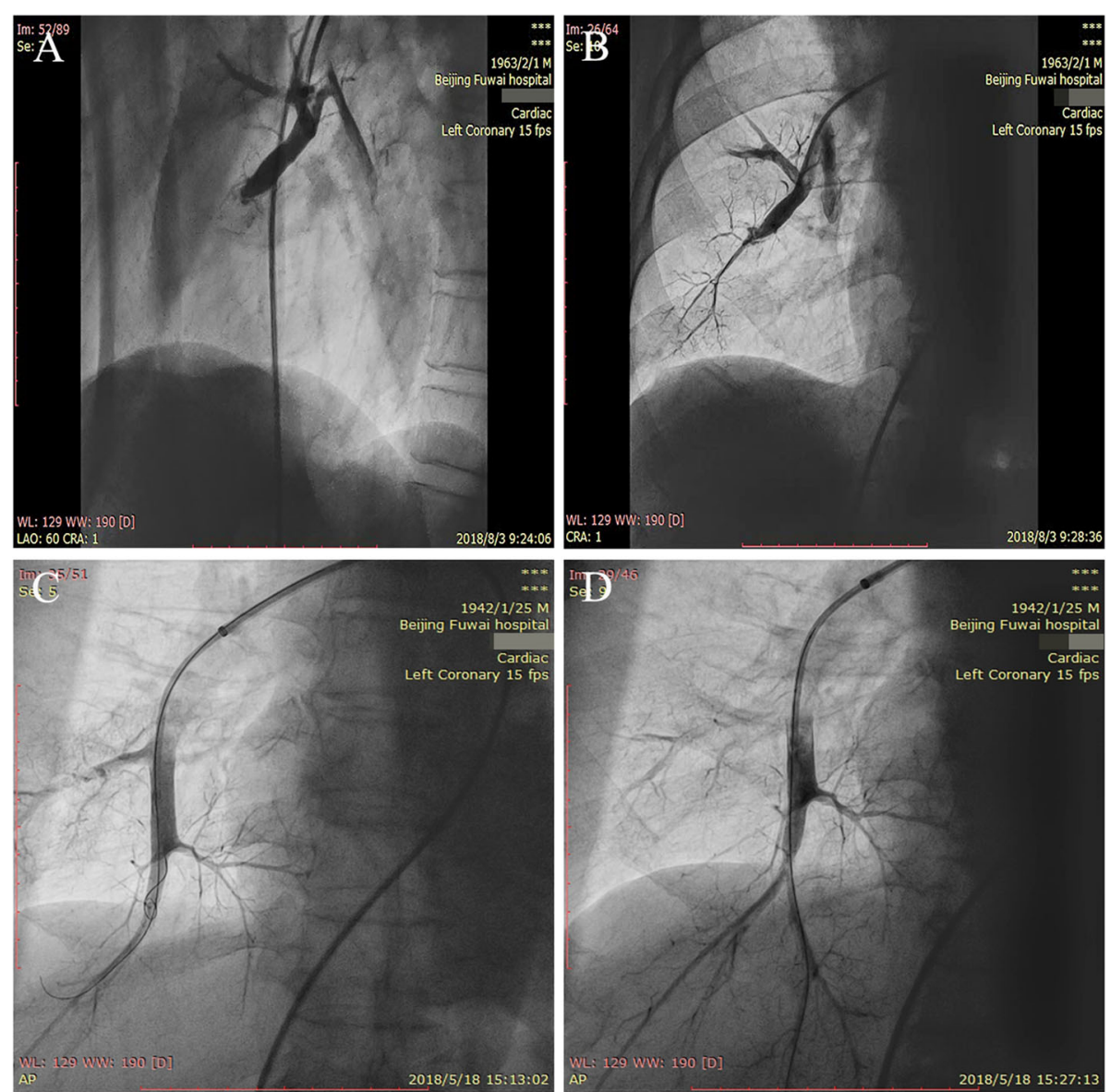

Fig. 1 Selective pulmonary angiography in right lower lobe before and immediately after BPA. a Total occlusion in RA8 before BPA. b Improved blood flow in RA8 immediately after BPA. c Total occlusion in RA10 before BPA. $\mathbf{d}$ Improved blood flow in RA10 immediately after BPA

evaluated by several CPET parameters improved significantly after BPA. Compared to pre-BPA, Peak WR $(78.0 \pm 40.6 \mathrm{w}$ vs $86.7 \pm 39.8 \mathrm{w}, P<0.001)$, Peak $\mathrm{VO}_{2}$ $(13.9 \pm 3.7 \mathrm{ml} / \mathrm{kg} / \mathrm{min}$ vs $15.2 \pm 3.3 \mathrm{ml} / \mathrm{kg} / \mathrm{min}, P<0.001)$, Peak $\mathrm{VO}_{2} \%$ predicted $(54.5 \pm 16.8 \%$ vs $58.8 \pm 15.3 \%, P<$ $0.001)$, peak respiratory exchange ratio $(1.01 \pm 0.08$ vs $1.06 \pm 0.10, P<0.001)$, OUES $(1250.5 \pm 508.3 \mathrm{ml} / \mathrm{min} /$ $1 / \mathrm{min}$ vs $1329.1 \pm 466.6 \mathrm{ml} / \mathrm{min} / \mathrm{l} / \mathrm{min}, P=0.015)$ and HR\% predicted $(78.5 \pm 10.5 \%$ vs $84.9 \pm 17.4 \%, P=$ 0.031 ) all significantly increased post-BPA. Similarly, notable increments were also identified in ventilation parameters VEmax $(47.2 \pm 14.4 \mathrm{l} / \mathrm{min}$ vs $50.7 \pm 15.1 \mathrm{l} /$ min, $P=0.027)$, VT@AT $(1.19 \pm 0.31 \mathrm{l}$ vs $1.31 \pm 0.34 \mathrm{l}$, $P=0.035)$ and VTmax $(1.54 \pm 0.38 \mathrm{l}$ vs $1.67 \pm 0.43 \mathrm{l}$, $P<0.001)$. HR@ AT $(102.8 \pm 18.3 \mathrm{bpm}$ vs $108.1 \pm 19.0$ bpm, $P=0.056)$ and Peak $\mathrm{VO}_{2} / \mathrm{HR} \%$ predicted $(69.0 \pm 17.9 \%$ vs $71.3 \pm 15.3 \%, P=0.068)$ before and after BPA reached a borderline statistical significance. $\mathrm{VE} / \mathrm{VCO}_{2}$ slope, $\mathrm{VE} / \mathrm{VCO}_{2}$ slope\% predicted and Peak $\mathrm{VE} / \mathrm{VCO}_{2}$ were on a downward trend, although no significant differences were found between preBPA and post-BPA.

\section{Hemodynamics and cardiopulmonary function after multiple BPA sessions}

For ten CTEPH patients receiving repeated BPA sessions (2-4 sessions), a subgroup analysis was performed. The median time interval between the first and last BPA sessions was 14.4 (9.8-29.2) weeks. As described in Table 2, $\mathrm{SaO}_{2}(90.2 \pm 1.8 \%$ vs $91.9 \pm 1.6 \%, P=0.01)$ and $\mathrm{SvO}_{2}$ $(68.14 \pm 4.43 \%$ vs $72.07 \pm 2.97 \%, P=0.019)$ were both significantly increased before the last BPA session when compared with those before the first BPA session. In addition, mPAP decreased from $52.6 \pm 14.1 \mathrm{mmHg}$ to $44.8 \pm 8.2 \mathrm{mmHg}(P=0.031$, Additional file 2: Figure S1), and PVR declined from $1067.0 \pm 410.4$ dyne.s. $\mathrm{cm}^{-5}$ to $758.0 \pm 212.1$ dyne $\cdot \mathrm{s} \cdot \mathrm{cm}^{-5}$, while CI remained unchanged. LVED measured by TTE was significantly elevated from $41.4 \pm 3.7 \mathrm{~mm}$ to $43.7 \pm 3.8 \mathrm{~mm}$. In contrast to data before first-BPA, all variables of PFT as was mentioned above augmented significantly after last-BPA. Furthermore, the average level of NT-proBNP fell significantly over time from $1329.0 \mathrm{pg} / \mathrm{ml}$ to $326.5 \mathrm{pg} / \mathrm{ml}$, while $6 \mathrm{MWD}$ rose to $443.9 \pm 63.6 \mathrm{~m}$ after the last BPA session (Additional file 2: Figure S1). Seven (70\%) 
Table 2 Hemodynamics and clinical parameters before and after a single BPA session and multiple BPA sessions

\begin{tabular}{|c|c|c|c|c|c|c|}
\hline \multirow[t]{2}{*}{ Parameters } & \multicolumn{3}{|c|}{ Single BPA session $(n=40)$} & \multicolumn{3}{|c|}{ Multiple BPA sessions $(n=10)$} \\
\hline & Pre-BPA & Post-BPA & $P$ value & Before-first BPA & After-last BPA & $P$ value \\
\hline \multicolumn{7}{|l|}{$\mathrm{RHC}$} \\
\hline $\mathrm{SvO}_{2}, \%$ & $69.8 \pm 5.1$ & - & & $68.14 \pm 4.43$ & $72.07 \pm 2.97^{\mathrm{a}}$ & 0.019 \\
\hline $\mathrm{SaO}_{2}, \%$ & $91.4 \pm 2.1$ & $91.9 \pm 2.7$ & 0.262 & $90.2 \pm 1.8$ & $91.9 \pm 1.6^{\mathrm{a}}$ & 0.01 \\
\hline $\mathrm{mRAP}, \mathrm{mmHg}$ & $7.4 \pm 2.9$ & - & & $7.3 \pm 3.9$ & $8.1 \pm 2.5^{\mathrm{a}}$ & 0.54 \\
\hline $\mathrm{sPAP}, \mathrm{mmHg}$ & $84.4 \pm 22.8$ & $74.7 \pm 21.9$ & $<0.001$ & $93.0 \pm 26.0$ & $81.2 \pm 18.7^{\mathrm{a}}$ & 0.068 \\
\hline $\mathrm{dPAP}, \mathrm{mmHg}$ & $29.4 \pm 7.8$ & $25.5 \pm 7.3$ & $<0.001$ & $31.9 \pm 9.0$ & $27.8 \pm 4.3^{\mathrm{a}}$ & 0.089 \\
\hline $\mathrm{mPAP}, \mathrm{mmHg}$ & $47.4 \pm 11.9$ & $41.5 \pm 10.7$ & $<0.001$ & $52.6 \pm 14.1$ & $44.8 \pm 8.2^{\mathrm{a}}$ & 0.031 \\
\hline PVR, dyne $\cdot s \cdot \mathrm{cm}^{-5}$ & $886.15 \pm 342.04$ & - & & $1067.0 \pm 410.4$ & $758.0 \pm 212.1^{\mathrm{a}}$ & 0.04 \\
\hline PAWP, mmHg & $9.8 \pm 3.2$ & - & & $8.1 \pm 2.8$ & $10.7 \pm 3.0^{\mathrm{a}}$ & 0.164 \\
\hline $\mathrm{Cl}, 1 / \mathrm{min} / \mathrm{m}^{2}$ & $3.26 \pm 0.78$ & - & & $3.07 \pm 0.69$ & $3.42 \pm 0.70^{\mathrm{a}}$ & 0.202 \\
\hline \multicolumn{7}{|l|}{ TTE } \\
\hline LVED, mm & $41.1 \pm 4.9$ & $42.0 \pm 5.1$ & 0.203 & $41.4 \pm 3.7$ & $43.7 \pm 3.8$ & 0.048 \\
\hline RVED, mm & $31.3 \pm 7.4$ & $30.2 \pm 6.4$ & 0.142 & $30.5 \pm 4.0$ & $28.1 \pm 5.1$ & 0.32 \\
\hline TAPSE, mm & $16.5 \pm 4.2$ & $17.2 \pm 3.5$ & 0.296 & $16.2 \pm 4.2$ & $18.3 \pm 1.5$ & 0.304 \\
\hline \multicolumn{7}{|c|}{ Pulmonary function testing } \\
\hline FVC, I & $3.11 \pm 0.83$ & $3.21 \pm 0.93$ & 0.037 & $3.22 \pm 0.84$ & $3.49 \pm 1.01$ & 0.01 \\
\hline FVC\% predicted, \% & $90.7 \pm 13.0$ & $93.4 \pm 14.5$ & 0.041 & $95.8 \pm 12.0$ & $103.6 \pm 11.2$ & 0.004 \\
\hline FEV1, I & $2.26 \pm 0.64$ & $2.32 \pm 0.71$ & 0.034 & $2.39 \pm 0.69$ & $2.55 \pm 0.79$ & 0.023 \\
\hline FEV1\% predicted, $\%$ & $80.8 \pm 14.2$ & $82.7 \pm 16.1$ & 0.045 & $87.9 \pm 16.5$ & $93.2 \pm 15.4$ & 0.038 \\
\hline $\mathrm{MW}, \mathrm{l} / \mathrm{min}$ & $89.4 \pm 31.7$ & $99.1 \pm 34.7$ & $<0.001$ & $95.2 \pm 37.7$ & $114.1 \pm 36.2$ & 0.002 \\
\hline MW\% predicted, \% & $83.2 \pm 24.0$ & $92.4 \pm 25.2$ & $<0.001$ & $89.0 \pm 26.4$ & $108.4 \pm 26.0$ & 0.005 \\
\hline NT-proBNP, pg/ml & $1161.8 \pm 1420.0$ & $671.2 \pm 753.0$ & 0.001 & $1329.0 \pm 1599.0$ & $326.5 \pm 601.0$ & 0.022 \\
\hline $6 \mathrm{MWD}, \mathrm{m}$ & $350.0 \pm 103.4$ & $403.6 \pm 81.3$ & $<0.001$ & $316.8 \pm 120.2$ & $443.9 \pm 63.6$ & 0.003 \\
\hline WHO-FC I/II/III/IV & $2.3 \pm 0.5$ & $1.9 \pm 0.5$ & $<0.001$ & $2.7 \pm 0.5$ & $2.0 \pm 0$ & 0.001 \\
\hline I, n (\%) & $1(2.5 \%)$ & $8(20 \%)$ & & $0(0 \%)$ & $0(0 \%)$ & \\
\hline$\|, \mathrm{n}(\%)$ & $26(65 \%)$ & 30 (75\%) & & $3(30 \%)$ & 10 (100\%) & \\
\hline III, n (\%) & $13(32.5 \%)$ & $2(5 \%)$ & & 7 (70\%) & $0(0 \%)$ & \\
\hline IV, n (\%) & $0(0 \%)$ & $0(0 \%)$ & & $0(0 \%)$ & $0(0 \%)$ & \\
\hline
\end{tabular}

$B P A$ balloon pulmonary angioplasty, $\mathrm{RHC}$ right heart catheterization, $\mathrm{SvO}_{2}$ mixed venous oxygen saturation, $\mathrm{SaO}_{2}$ arterial oxygen saturation, $m R A P$ mean right atrial pressure, $S P A P$ systolic pulmonary artery pressure, $A P A P$ diastolic pulmonary artery pressure, mPAP mean pulmonary artery pressure, $P V R$ pulmonary vascular resistance, $P A W P$ pulmonary arterial wedge pressure, $C I$ cardiac index, $T E E$ transthoracic echocardiography, $L V E D$ left ventricular end-diastolic diameter, RVED right ventricular end-diastolic diameter, TAPSE tricuspid annular plane systolic excursion, FVC forced vital capacity, FEV1 forced expiratory volume in the first second, $M V V$ maximal voluntary ventilation, NT-proBNP N-terminal pro-brain natriuretic peptide, 6MWD 6-min walk distance, WHO-FC WHO functional class ${ }^{\mathrm{a}}$ Data at RHC before the last BPA

patients in WHO-FC III prior to the first BPA session improved to WHO-FC II after the final procedure. The changes of CPET parameters before-first and after-last BPA sessions were displayed in Table 3. Compared with baseline, Peak WR, Peak $\mathrm{VO}_{2}$, Peak $\mathrm{VO}_{2} \%$ predicted, peak respiratory exchange ratio and $\mathrm{HR} \%$ predicted were still obviously elevated (Additional file 2: Figure S2). HRmax during exercise increased significantly from $126.4 \pm 17.0 \mathrm{bpm}$ to $141.8 \pm 13.0 \mathrm{bpm}(P<0.001)$, yet differences of VTmax between before-first BPA and afterlast BPA reached a marginal significance $(P=0.051)$. Non-significant increased trends in OUES, Peak $\mathrm{VO}_{2} /$ $\mathrm{HR}$ and $\mathrm{SpO}_{2}$ were discerned.

\section{Discussion}

This is, to the best of our knowledge, the first study to investigate the safety and efficacy of BPA in China, as well as its instant effects on cardiopulmonary function in patients with inoperable CTEPH. The present study demonstrated BPA, as a safe and effective approach, brought instant and lasting benefits to hemodynamics and cardiopulmonary function for patients with inoperable CTEPH.

PEA is currently the only established curative treatment for patients with CTEPH [14]. Nevertheless, nearly $40 \%$ of subjects were considered inoperable because of lesion inaccessbility or possible comorbidities $[4,8]$, and 

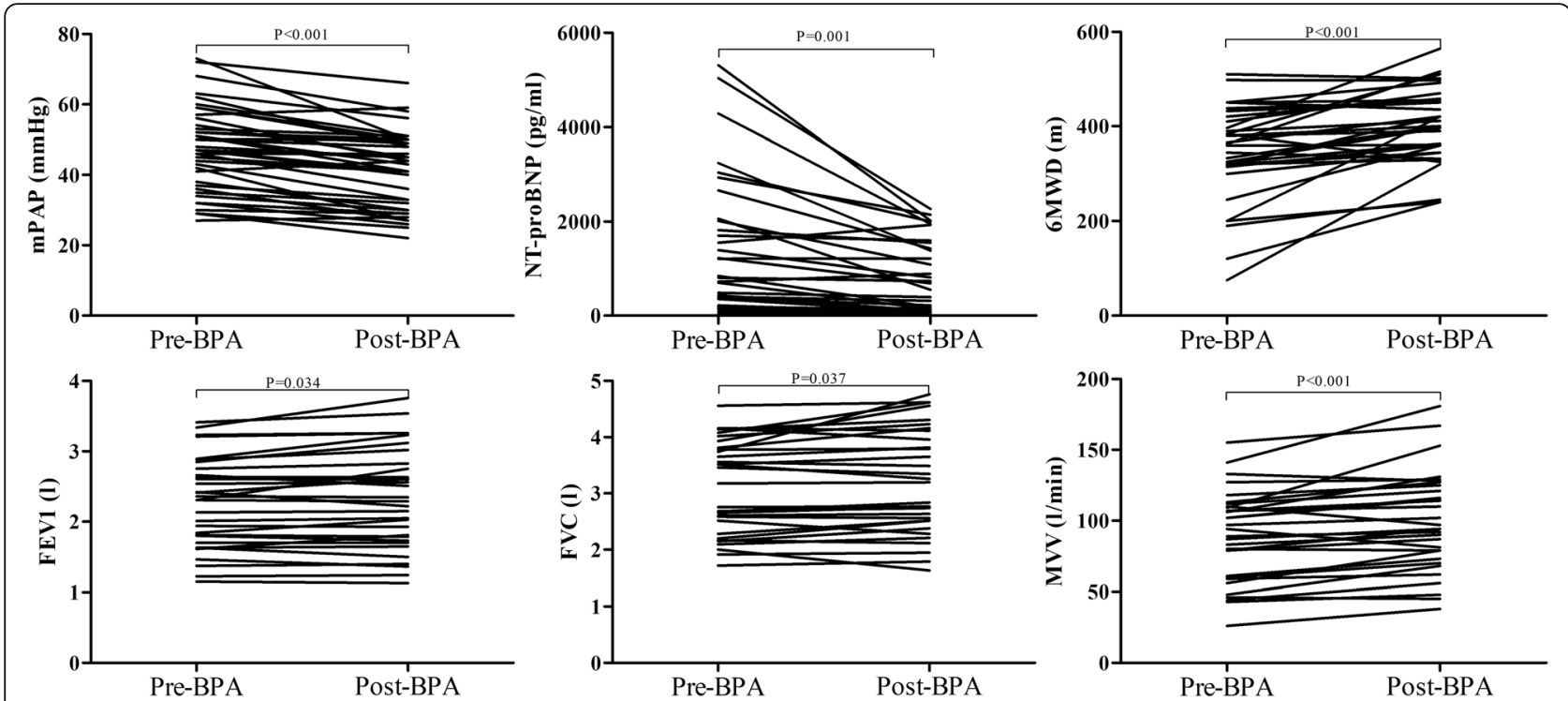

Fig. 2 Hemodynamic and clinical parameters before and after a single BPA session

Table 3 CPET parameters before and after a single BPA session and multiple BPA sessions

\begin{tabular}{|c|c|c|c|c|c|c|}
\hline \multirow[t]{2}{*}{ Parameters } & \multicolumn{3}{|c|}{ Single BPA session $(n=40)$} & \multicolumn{3}{|c|}{ Multiple BPA sessions $(n=10)$} \\
\hline & Pre-BPA & Post-BPA & $P$ value & Before-first BPA & After-last BPA & $P$ value \\
\hline Peak WR, w & $78.0 \pm 40.6$ & $86.7 \pm 39.8$ & $<0.001$ & $77.3 \pm 47.6$ & $96.2 \pm 40.2$ & 0.024 \\
\hline AT, $\mathrm{ml} / \mathrm{kg} / \mathrm{min}$ & $10.7 \pm 3.2$ & $11.7 \pm 3.1$ & 0.098 & $10.3 \pm 3.2$ & $11.4 \pm 3.3$ & 0.57 \\
\hline Peak $\mathrm{VO}_{2}, \mathrm{ml} / \mathrm{kg} / \mathrm{min}$ & $13.9 \pm 3.7$ & $15.2 \pm 3.3$ & $<0.001$ & $13.7 \pm 4.5$ & $16.1 \pm 3.2$ & 0.025 \\
\hline Peak $\mathrm{VO}_{2} \%$ predicted, $\%$ & $54.5 \pm 16.8$ & $58.8 \pm 15.3$ & $<0.001$ & $53.7 \pm 18.1$ & $63.9 \pm 14.1$ & 0.015 \\
\hline Peak respiratory exchange ratio & $1.01 \pm 0.08$ & $1.06 \pm 0.10$ & $<0.001$ & $1.04 \pm 0.08$ & $1.09 \pm 0.10$ & 0.046 \\
\hline VENCO 2 slope & $49.1 \pm 15.3$ & $45.3 \pm 11.4$ & 0.09 & $52.3 \pm 16.7$ & $44.0 \pm 10.5$ & 0.099 \\
\hline VENCO 2 slope\% predicted, $\%$ & $181.4 \pm 57.0$ & $166.7 \pm 40.6$ & 0.079 & $191.4 \pm 62.7$ & $160.7 \pm 37.8$ & 0.098 \\
\hline OUES, $\mathrm{ml} / \mathrm{min} / 1 / \mathrm{min}$ & $1250.5 \pm 508.3$ & $1329.1 \pm 466.6$ & 0.015 & $1205.0 \pm 576.2$ & $1341.0 \pm 382.6$ & 0.397 \\
\hline VEmax, $1 / \min$ & $47.2 \pm 14.4$ & $50.7 \pm 15.1$ & 0.027 & $52.4 \pm 17.1$ & $56.8 \pm 15.4$ & 0.197 \\
\hline VT@AT, I & $1.19 \pm 0.31$ & $1.31 \pm 0.34$ & 0.035 & $1.20 \pm 0.37$ & $1.31 \pm 0.33$ & 0.422 \\
\hline VTmax, I & $1.54 \pm 0.38$ & $1.67 \pm 0.43$ & $<0.001$ & $1.66 \pm 0.39$ & $1.80 \pm 0.45$ & 0.051 \\
\hline HR@AT, bpm & $102.8 \pm 18.3$ & $108.1 \pm 19.0$ & 0.056 & $102.3 \pm 15.1$ & $108.6 \pm 14.4$ & 0.388 \\
\hline HRmax, bpm & $128.9 \pm 19.5$ & $132.9 \pm 16.0$ & 0.16 & $126.4 \pm 17.0$ & $141.8 \pm 13.0$ & $<0.001$ \\
\hline HR\% predicted, \% & $78.5 \pm 10.5$ & $84.9 \pm 17.4$ & 0.031 & $79.7 \pm 9.8$ & $88.7 \pm 7.5$ & $<0.001$ \\
\hline Peak $\mathrm{VO}_{2} / \mathrm{HR}, \mathrm{ml} / \mathrm{bpm}$ & $7.52 \pm 2.88$ & $7.66 \pm 2.55$ & 0.381 & $7.24 \pm 3.37$ & $7.73 \pm 2.49$ & 0.466 \\
\hline Peak $\mathrm{VO}_{2} / \mathrm{HR} \%$ predicted, $\%$ & $69.0 \pm 17.9$ & $71.3 \pm 15.3$ & 0.068 & $66.6 \pm 18.9$ & $71.8 \pm 12.0$ & 0.272 \\
\hline Peak PetCO, $\mathrm{mmHg}$ & $24.7 \pm 6.3$ & $24.6 \pm 7.0$ & 0.916 & $22.4 \pm 5.3$ & $24.4 \pm 4.9$ & 0.39 \\
\hline Peak PetO $2, \mathrm{mmHg}$ & $125.0 \pm 5.0$ & $125.7 \pm 4.9$ & 0.253 & $126.8 \pm 3.8$ & $127.8 \pm 3.8$ & 0.454 \\
\hline Peak VENO 2 & $52.3 \pm 12.6$ & $51.6 \pm 10.6$ & 0.609 & $60.1 \pm 14.4$ & $54.4 \pm 9.6$ & 0.359 \\
\hline Peak VE $/ \mathrm{NCO}_{2}$ & $51.8 \pm 12.9$ & $49.6 \pm 10.7$ & 0.078 & $57.9 \pm 13.5$ & $50.5 \pm 10.5$ & 0.19 \\
\hline Peak $\mathrm{SpO}_{2}, \%$ & $91.0 \pm 5.1$ & $92.4 \pm 4.7$ & 0.484 & $89.1 \pm 4.6$ & $90.9 \pm 4.1$ & 0.213 \\
\hline
\end{tabular}

Peak WR peak work rate, $A T$ anaerobic threshold, $P e a k V_{2}$ peak oxygen consumption, $V E N V_{2} O_{2}$ minute ventilation/carbon dioxide production, OUES oxygen uptake efficiency slope, VEmax maximal minute ventilation, VT@AT tidal volume at the anaerobic threshold, VTmax maximum tidal volume, HR@AT heart rate at the anaerobic threshold, $\mathrm{HRmax}$ maximum heart rate, Peak $\mathrm{VO}_{2} / \mathrm{HR}$ peak oxygen consumption/peak heart rate, $\mathrm{PetCO}_{2}$ partial pressure of end-tidal carbon dioxide, $\mathrm{PetO}_{2}$ partial pressure of end-tidal oxygen, $\mathrm{VE} / \mathrm{O}_{2}$ minute ventilation/oxygen consumption, $\mathrm{SpO}_{2}$ pulse oxygen saturation 

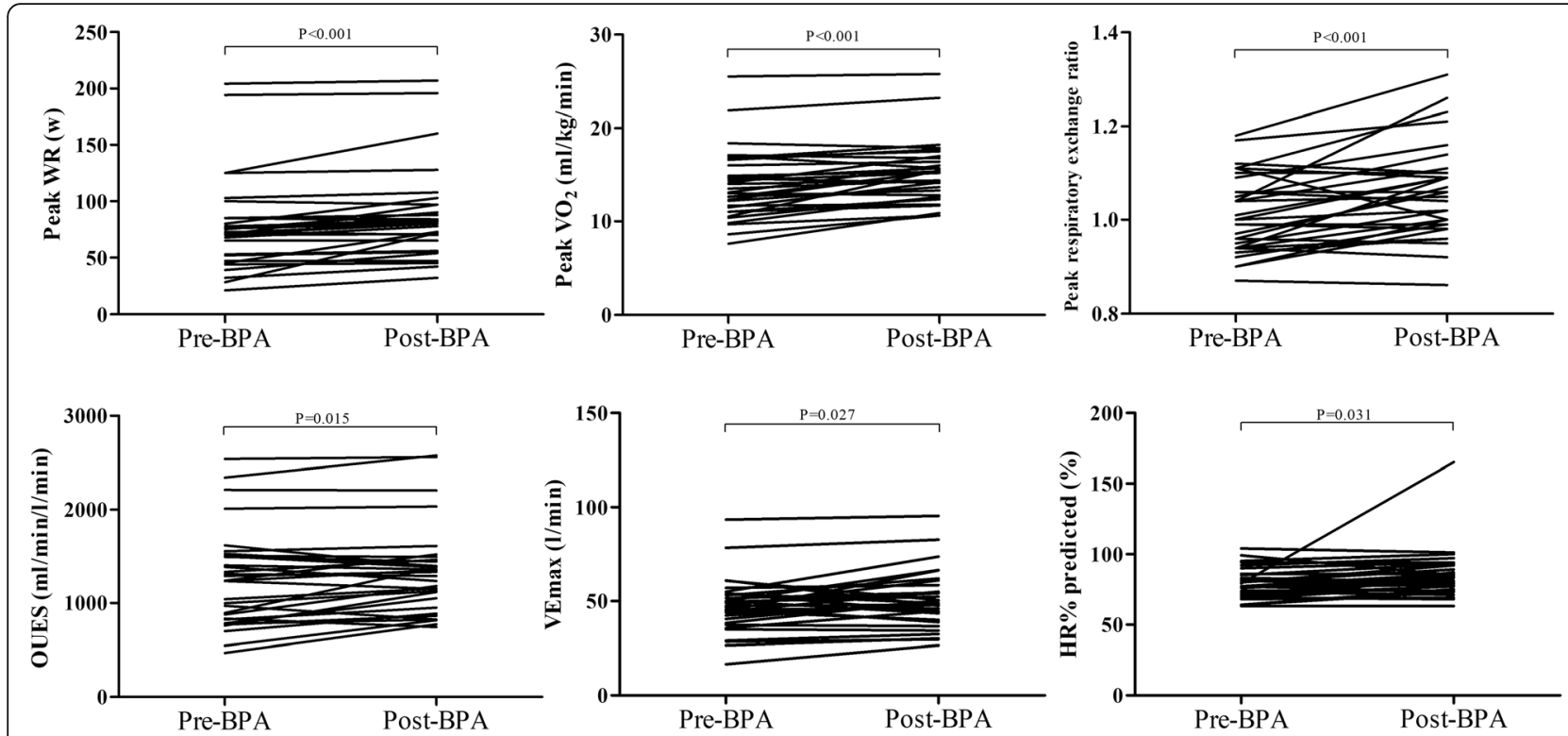

Fig. 3 CPET parameters before and after a single BPA session

25-31\% of CTEPH patients had residual PH after PEA surgery $[27,28]$. Moreover, PEA surgery involves exquisite techniques and multidisciplinary collaboration, and can be performed only at a limited number of institutions. Riociguat, a soluble guanylate cyclase stimulator, is the only approved medical therapy indicated for patients with inoperable CTEPH based on the CHEST trials $[6,29,30]$. However, majorities of CTEPH patients cannot benefit from this drug for economic reasons in China.

BPA is an interventional technique which uses a balloon catheter to cross and dilate target lesions to relieve pulmonary vascular stenosis or obstruction. It was firstly attempted in 1983 to relieve pulmonary artery stenosis or hypoplasia, and brought significant hemodynamic improvements for children with congenital lesions [31]. The first case of BPA for CTEPH was reported by Voorburg et al. in 1988 [32]. Later in 2001, Feinstein described 18 CTEPH case series treated with BPA, showing improved hemodynamics and exercise tolerance. Unfortunately, 11 patients developed reperfusion pulmonary edema, and 3 patients required mechanical ventilation [10]. With continuous refinements from three Japanese institutions [11-13], BPA has been reported to significantly improve hemodynamics, exercise capacity and right ventricular function, with significantly lower rates of complications [33-36]. BPA is now recommended in the newly proposed CTEPH treatment algorithm for inoperable CTEPH patients at experienced centers [4], however, most of data with regard to BPA in CTEPH mainly come from Japan, Europe and USA, the safety and efficacy of BPA procedures in China have never been reported.
Our study demonstrated that BPA conferred significant improvements in instant hemodynamics as evidenced by decreased sPAP, dPAP and mPAP. However, differential structural changes in the heart evaluated by TTE were not observed after a single BPA session among our patients. In accordance with our results, Tsugu et al. [33] also noticed mPAP was significantly improved, and right ventricular basal, mid cavity and longitudinal diameters remained unchanged immediately after BPA, but left ventricular end-diastolic diameter and TAPSE obviously increased compared with baseline, the difference might be explained by relatively better right ventricular function and higher TAPSE of our patients. In order to exclude the effect of supplemental oxygen on pulmonary hemodynamics during BPA procedures, we compared catheterization hemodynamics before the first $\mathrm{BPA}$ and before the second BPA among patients receiving multiple BPA sessions which revealed mPAP decreased from $52.6 \pm 14.1 \mathrm{mmHg}$ to $44.5 \pm 9.2 \mathrm{mmHg}$ $(P=0.004)$ and PVR declined from $1067.0 \pm 410.4$ dyne $\cdot \mathrm{s} \cdot \mathrm{cm}^{-5}$ to $822.6 \pm 272.4$ dyne.s $\cdot \mathrm{cm}^{-5} \quad(P=0.025)$, definitely demonstrating a single BPA session was enough to improve hemodynamics. Except for hemodynamics, PFT indexes, 6MWD, NT-proBNP and WHO-FC all got improved after a single BPA session.

Those receiving multiple BPA sessions got lasting hemodynamic improvements as evidenced by reduced mPAP and PVR together with elevated oxygen saturation before the last BPA session when compared with baseline values. However, CI did not show any significant changes but presented a rising trend after BPA sessions among our patients, while previous studies 
reported significant improvements in CI [20, 34, 37], which might be ascribed to limited sessions, short BPA intervals and small sample size of our study. Most importantly, LVED got significantly increased, reflecting structural recovery. The above hemodynamic and structural changes are only based on 2-4 BPA sessions, we believe there will be a more significant improvement after more BPA sessions. Furthermore, 6MWD and NTproBNP got larger scale changes than those in a single BPA session, signifying more benefits brought by staged BPA sessions.

Previous studies reported the rates of lung injury, hemoptysis, and pulmonary artery perforation were 7.0$31.4 \%, 5.6-19.6 \%$, and $0-8.0 \%$ respectively [35]. Occurrence of complications depends on target lesion types, guiding wire types, balloon size and interventionist experience, too many target vessels dilated per session or too large balloon are easy to cause aforementioned complications. No such complications occurred among our patients, we utilized pulmonary angiography and pulmonary perfusion scintigraphy before BPA procedures to facilitate the selection of target lesions, preferred to select smaller balloons $(2 \mathrm{~mm})$ at the initial BPA session no matter what lesions our patients had, and limited the total number of balloons (1-2 per session). As subtotal occlusion, total occlusion and tortuous lesions were accompanied with higher risks of complications such as wire injury [38], we started with "webs and bands" and performed balloon dilatation in a staged manner, indicating a relatively conservative approach may lower BPA complications.

CPET is an important clinical tool to evaluate exercise capacity and cardiopulmonary function. Peak $\mathrm{VO}_{2}$, an essential factor that mainly reflects exercise capacity and cardiac function, is currently an important index of risk stratification in patients with PAH $[39,40]$. In patients with inoperable CTEPH, Peak $\mathrm{VO}_{2}$ is still a powerful predictor of mortality [41]. After a median 14.4-week interval between the first and last BPA, Peak $\mathrm{VO}_{2}$ and Peak $\mathrm{VO}_{2} \%$ predicted both strikingly increased among our patients receiving multiple BPA sessions, indicating a significantly lasting recovery in exercise capacity and cardiac function and possible improvement of prognosis, other similar parameters such as peak respiratory exchange ratio (respiratory quotient), HRmax and HR\% predicted were also comparable to those before $\mathrm{BPA}$, which is consistent with previous studies $[20,34] . \mathrm{VE} / \mathrm{VCO}_{2}$ slope, a sensitive variable that reflects exercise ventilatory efficiency, decreased greatly even in the early 1 month after PEA [42] as well as 3 months after BPA sessions [34]. However, $\mathrm{VE} / \mathrm{VCO}_{2}$ slope showed a non-significantly declining tendency, which might be influenced by small population size.
Unlike earlier studies in which cardiopulmonary function was assessed late after interventions [20, 34], our study mainly focused on the early changes of hemodynamics and cardiopulmonary function within a week after BPA procedure, thus revealing immediate effectiveness of BPA. Peak WR, Peak $\mathrm{VO}_{2}$, Peak $\mathrm{VO}_{2} \%$ predicted significantly improved when assessed within 3 days following a single BPA session. OUES is a submaximal exercise parameter that represents the absolute rate of increase in oxygen consumption per 10-fold increase in ventilation, our previous study demonstrated OUES could predict poor outcome in patients with idiopathic PAH [25]. Herein, we observed early increase of OUES after a single BPA session, indicating instant improvement of submaximal exercise capacity. VE and VT are two good indicators of ventilation function, VEmax and VTmax were both evidently elevated, illustrating early ventilation improvements. FVC, FEV1, and MVV have been widely used clinically to evaluate pulmonary function in respiratory diseases, they all achieved instant and lasting correction after BPA procedure, which may be explained by ventilation improvement along with increased pulmonary blood flow so as to maintain the dynamic balance of ventilation and perfusion. In consistent with aforementioned ventilation improvement, CTEPH patients felt much better and breathed more deeper and easier even during BPA procedures in our real-world clinical practice. CPET could objectively, safely and repetitiously evaluate the cardiopulmonary function, exhibiting absolute advantages over subjective parameters such as WHO-FC and 6MWD as well as catherization hemodynamics which might be confounded by supplemental oxygen during BPA, CPET may exert as a sensitive and reliable indicator to assess the effects of BPA.

There are several limitations of this study. First, the sample size of this study is relatively small. Second, this study was implemented in a single center, although our center is the largest pulmonary vascular center and also one of the largest BPA centers in China. Large-scale, multicenter, prospective studies should be launched to investigate and confirm the long-term effects of BPA procedures for CTEPH patients. Third, we didn't measure other parameters such as oxygen saturation after BPA for economic issues and procedure time, thus immediate PVR and CI were not obtained, leading to incomprehensive hemodynamics evaluation after a single BPA session.

\section{Conclusion}

BPA can safely and effectively improve the instant and lasting hemodynamics and cardiopulmonary function. Larger multicenter studies are needed to further investigate and confirm the role of BPA in CTEPH. 


\section{Supplementary information}

Supplementary information accompanies this paper at https://doi.org/10. 1186/s12931-019-1211-y.

Additional file 1. Representative videos before and immediately after BPA. Additional file 2: Figure S1. Hemodynamic and clinical parameters before and after multiple BPA sessions. Figure S2. CPET parameters before and after multiple BPA sessions.

\section{Abbreviations}

6MWD: 6-min walk distance; AT: Anaerobic threshold; BPA: Balloon pulmonary angioplasty; Cl: Cardiac index; CPET: Cardiopulmonary exercise testing; CTEPH: Chronic thromboembolic pulmonary hypertension; DVT: Deep vein thrombosis; FEV1: Forced expiratory volume in the first second; FVC: Forced vital capacity; LVED: Left ventricular end-diastolic diameter; MPAP: Mean pulmonary arterial pressure; MW: Maximal voluntary ventilation; NT-proBNP: N-terminal pro-brain natriuretic peptide: OUES: Oxygen uptake efficiency slope; PAH: Pulmonary arterial hypertension. PAWP: Pulmonary arterial wedge pressure; PEA: Pulmonary endarterectomy; PH: Pulmonary hypertension; PVR: Pulmonary vascular resistance; RAP: Right atrium pressure; RHC: Right heart catheterization; RVED: Right ventricular end-diastolic diameter; SaO2: Oxygen saturation; SvO2: Mixed venous oxygen saturation; TAPSE: Tricuspid annular plane systolic excursion;

TTE: Transthoracic echocardiography; VE: Minute ventilation; WHO-FC: World Health Organization functional class; WR: Work rate

\section{Acknowledgements}

We thank Professor Hiromi Matsubara, Department of Clinical Science, National Hospital Organization Okayama Medical Center, Okayama, Japan, for his direction in BPA procedures.

\section{Authors' contributions}

JQ contributed to the conception and design of the study, analysis and interpretation of data, drafting and revising the manuscript. LQ, YT, ZQX and $\mathrm{ZZH}$ contributed to data collection and interpretation. JQ, YX, YL, ZY, ZQ, MXP and ACH contributed to data collection. XCM critically reviewed and revised the manuscript. $\mathrm{ZZH}$ and $\mathrm{LZH}$ conceived and designed the study, and helped revise the manuscript. All authors read and approved the final manuscript.

\section{Funding}

This paper is supported by National Natural Science Foundation of China (Grant Nos. 81370326, 81641005), Beijing Municipal Science and Technology Project (Z181100001718200) and National Precision Medical Research Program of China (2016YFC0905602)

\section{Availability of data and materials}

All data generated or analyzed during this study are included in this manuscript.

\section{Ethics approval and consent to participate}

The study was approved by the ethics committee of Fuwai Hospital and adhered to the Declaration of Helsinki.

\section{Consent for publication}

Written informed consent was obtained from patients in this study.

\section{Competing interests}

The authors declare that they have no competing interests.

Received: 17 June 2019 Accepted: 10 October 2019

Published online: 08 November 2019

\section{References}

1. Hoeper MM, Mayer E, Simonneau G, Rubin LJ. Chronic thromboembolic pulmonary hypertension. Circulation. 2006;113:2011-20.

2. Kim NH, Delcroix M, Jenkins DP, Channick R, Dartevelle P, Jansa P, Lang I, Madani MM, Ogino H, Pengo V, Mayer E. Chronic thromboembolic pulmonary hypertension. J Am Coll Cardiol. 2013;62:D92-9.
3. Mahmud E, Madani MM, Kim NH, Poch D, Ang L, Behnamfar O, Patel MP, Auger WR. Chronic thromboembolic pulmonary hypertension: evolving therapeutic approaches for operable and inoperable disease. J Am Coll Cardiol. 2018;71:2468-86.

4. Kim NH, Delcroix M, Jais X, Madani MM, Matsubara H, Mayer E, Ogo T, Tapson VF, Ghofrani HA, Jenkins DP. Chronic thromboembolic pulmonary hypertension. Eur Respir J. 2019;53:1801915.

5. Yan L, Li X, Liu Z, Zhao Z, Luo Q, Zhao Q, Jin Q, Yu X, Zhang Y. Research progress on the pathogenesis of CTEPH. Heart Fail Rev. 2019. https://doi. org/10.1007/s10741-019-09802-4.

6. Zhang Y, Yu X, Jin Q, Luo Q, Zhao Z, Zhao Q, Yan L, Liu Z. Advances in targeted therapy for chronic thromboembolic pulmonary hypertension. Heart Fail Rev. 2019. https://doi.org/10.1007/s10741-019-09798-X.

7. Taniguchi $Y$, Jais $X$, Jevnikar M, Boucly A, Weatherald J, Brenot $P$, Planche $O$, Parent $F$, Savale $L$, Fadel $E$, et al. Predictors of survival in patients with notoperated chronic thromboembolic pulmonary hypertension. J Heart Lung Transplant. 2019;38:833-42.

8. Pepke-Zaba J, Delcroix M, Lang I, Mayer E, Jansa P, Ambroz D, Treacy C, D'Armini AM, Morsolini M, Snijder R, et al. Chronic thromboembolic pulmonary hypertension (CTEPH): results from an international prospective registry. Circulation. 2011;124:1973-81.

9. Yandrapalli S, Tariq S, Kumar J, Aronow WS, Malekan R, Frishman WH, Lanier GM. Chronic thromboembolic pulmonary hypertension: epidemiology, diagnosis, and management. Cardiol Rev. 2018;26:62-72.

10. Feinstein JA, Goldhaber SZ, Lock JE, Ferndandes SM, Landzberg MJ. Balloon pulmonary angioplasty for treatment of chronic thromboembolic pulmonary hypertension. Circulation. 2001;103:10-3.

11. Sugimura K, Fukumoto Y, Satoh K, Nochioka K, Miura Y, Aoki T, Tatebe S, Miyamichi-Yamamoto S, Shimokawa H. Percutaneous transluminal pulmonary angioplasty markedly improves pulmonary hemodynamics and long-term prognosis in patients with chronic thromboembolic pulmonary hypertension. Circ J. 2012;76:485-8.

12. Kataoka M, Inami T, Hayashida K, Shimura N, Ishiguro H, Abe T, Tamura Y, Ando M, Fukuda K, Yoshino H, Satoh T. Percutaneous transluminal pulmonary angioplasty for the treatment of chronic thromboembolic pulmonary hypertension. Circ Cardiovasc Interv. 2012;5:756-62.

13. Mizoguchi $H$, Ogawa A, Munemasa M, Mikouchi $H$, Ito $H$, Matsubara $H$. Refined balloon pulmonary angioplasty for inoperable patients with chronic thromboembolic pulmonary hypertension. Circ Cardiovasc Interv. 2012;5:748-55.

14. Galie N, Humbert M, Vachiery JL, Gibbs S, Lang I, Torbicki A, Simonneau G, Peacock A, Vonk Noordegraaf A, Beghetti M, et al. 2015 ESC/ERS Guidelines for the diagnosis and treatment of pulmonary hypertension: The Joint Task Force for the Diagnosis and Treatment of Pulmonary Hypertension of the European Society of Cardiology (ESC) and the European Respiratory Society (ERS): Endorsed by: Association for European Paediatric and Congenital Cardiology (AEPC), International Society for Heart and Lung Transplantation (ISHLT). Eur Respir J. 2015;46:903-75.

15. Albouaini K, Egred M, Alahmar A, Wright DJ. Cardiopulmonary exercise testing and its application. Postgrad Med J. 2007:83:675-82.

16. Zhao ZH, Liu ZH, Gu Q, Luo Q, Zhao Q, Xiong CM, Ni XH. Application of cardiopulmonary exercise testing in patients with chronic thromboembolic pulmonary hypertension. Zhonghua Yi Xue Za Zhi. 2013;93:1687-90.

17. Xi Q, Zhao Z, Liu Z, Ma X, Luo Q, Liu W. The lowest VENCO(2) ratio best identifies chronic thromboembolic pulmonary hypertension. Thromb Res. 2014;134:1208-13.

18. Condliffe R, Kiely DG, Gibbs JS, Corris PA, Peacock AJ, Jenkins DP, Goldsmith K, Coghlan JG, Pepke-Zaba J. Prognostic and aetiological factors in chronic thromboembolic pulmonary hypertension. Eur Respir J. 2009;33:332-8.

19. Schwaiblmair M, Faul C, von Scheidt W, Berghaus TM. Ventilatory efficiency testing as prognostic value in patients with pulmonary hypertension. BMC Pulm Med. 2012;12:23.

20. Andreassen AK, Ragnarsson A, Gude E, Geiran O, Andersen R. Balloon pulmonary angioplasty in patients with inoperable chronic thromboembolic pulmonary hypertension. Heart. 2013;99:1415-20.

21. Akizuki M, Serizawa N, Ueno A, Adachi T, Hagiwara N. Effect of balloon pulmonary angioplasty on respiratory function in patients with chronic thromboembolic pulmonary hypertension. Chest. 2017;151:643-9.

22. Yu X, Huang Z, Zhang Y, Liu Z, Luo Q, Zhao Z, Zhao Q, Gao L, Jin Q, Yan L. Obstructive sleep apnea in patients with chronic thromboembolic pulmonary hypertension. J Thorac Dis. 2018;10:5804-12. 
23. Xi Q, Liu Z, Liu W, Zhao Z, Luo Q, Huang Z. Chronic thromboembolic pulmonary hypertension is not associated with iron overload. Cardiovasc Pathol. 2015;24:76-9.

24. Yu X, Luo Q, Liu Z, Zhao Z, Zhao Q, An C, Huang Z, Jin Q, Gao L, Yan L. Prevalence of iron deficiency in different subtypes of pulmonary hypertension. Heart Lung. 2018;47:308-13.

25. Tang Y, Luo Q, Liu Z, Ma X, Zhao Z, Huang Z, Gao L, Jin Q, Xiong C, Ni X. Oxygen uptake efficiency slope predicts poor outcome in patients with idiopathic pulmonary arterial hypertension. J Am Heart Assoc. 2017;6: e005037.

26. Tang Y, Yao L, Liu Z, Xie W, Ma X, Luo Q, Zhao Z, Huang Z, Gao L, Jin Q, et al. Peak circulatory power is a strong prognostic factor in patients with idiopathic pulmonary arterial hypertension. Respir Med. 2018;135:29-34.

27. Freed DH, Thomson BM, Berman M, Tsui SS, Dunning J, Sheares KK, Pepke-Zaba J, Jenkins DP. Survival after pulmonary thromboendarterectomy: effect of residual pulmonary hypertension. J Thorac Cardiovasc Surg. 2011;141:383-7.

28. Hsieh WC, Jansa P, Huang WC, Niznansky M, Omara M, Lindner J. Residual pulmonary hypertension after pulmonary endarterectomy: a meta-analysis. J Thorac Cardiovasc Surg. 2018;156:1275-87.

29. Ghofrani HA, D'Armini AM, Grimminger F, Hoeper MM, Jansa P, Kim NH, Mayer E, Simonneau G, Wilkins MR, Fritsch A, et al. Riociguat for the treatment of chronic thromboembolic pulmonary hypertension. N Engl J Med. 2013;369:319-29.

30. Simonneau G, D'Armini AM, Ghofrani HA, Grimminger F, Jansa P, Kim NH, Mayer E, Pulido T, Wang C, Colorado P, et al. Predictors of long-term outcomes in patients treated with riociguat for chronic thromboembolic pulmonary hypertension: data from the CHEST-2 open-label, randomised, long-term extension trial. Lancet Respir Med. 2016;4:372-80.

31. Lock JE, Castaneda-Zuniga WR, Fuhrman BP, Bass JL. Balloon dilation angioplasty of hypoplastic and stenotic pulmonary arteries. Circulation. 1983:67:962-7.

32. Voorburg JA, Cats VM, Buis B, Bruschke AV. Balloon angioplasty in the treatment of pulmonary hypertension caused by pulmonary embolism. Chest. 1988:94:1249-53.

33. Tsugu T, Murata M, Kawakami T, Minakata Y, Kanazawa H, Kataoka M, Endoh J, Tsuruta H, Itabashi Y, Maekawa Y, et al. Changes in right ventricular dysfunction after balloon pulmonary angioplasty in patients with chronic thromboembolic pulmonary hypertension. Am J Cardiol. 2016;118:1081-7.

34. Fukui S, Ogo T, Goto Y, Ueda J, Tsuji A, Sanda Y, Kumasaka R, Arakawa T, Nakanishi M, Fukuda T, et al. Exercise intolerance and ventilatory inefficiency improve early after balloon pulmonary angioplasty in patients with inoperable chronic thromboembolic pulmonary hypertension. Int J Cardiol. 2015;180:66-8.

35. Tanabe N, Kawakami T, Satoh T, Matsubara H, Nakanishi N, Ogino H, Tamura Y, Tsujino I, Ogawa A, Sakao S, et al. Balloon pulmonary angioplasty for chronic thromboembolic pulmonary hypertension: a systematic review. Respir Investig. 2018;56:332-41.

36. Khan MS, Amin E, Memon MM, Yamani N, Siddiqi TJ, Khan SU, Murad MH, Mookadam F, Figueredo VM, Doukky R, et al. Meta-analysis of use of balloon pulmonary angioplasty in patients with inoperable chronic thromboembolic pulmonary hypertension. Int J Cardiol. 2019;291:134-9.

37. Araszkiewicz A, Darocha S, Pietrasik A, Pietura R, Jankiewicz S, Banaszkiewicz M, Slawek-Szmyt S, Biederman A, Mularek-Kubzdela T, Lesiak M, et al. Balloon pulmonary angioplasty for the treatment of residual or recurrent pulmonary hypertension after pulmonary endarterectomy. Int J Cardiol. 2019:278:232-7.

38. Kawakami T, Ogawa A, Miyaji K, Mizoguchi H, Shimokawahara H, Naito T, Oka T, Yunoki K, Munemasa M, Matsubara H. Novel angiographic classification of each vascular lesion in chronic thromboembolic pulmonary hypertension based on selective angiogram and results of balloon pulmonary angioplasty. Circ Cardiovasc Interv. 2016;9:e003318.

39. Wensel R, Opitz CF, Anker SD, Winkler J, Hoffken G, Kleber FX, Sharma R, Hummel M, Hetzer R, Ewert R. Assessment of survival in patients with primary pulmonary hypertension: importance of cardiopulmonary exercise testing. Circulation. 2002;106:319-24.

40. Wensel R, Francis DP, Meyer FJ, Opitz CF, Bruch L, Halank M, Winkler J, Seyfarth HJ, Glaser S, Blumberg F, et al. Incremental prognostic value of cardiopulmonary exercise testing and resting haemodynamics in pulmonary arterial hypertension. Int J Cardiol. 2013;167:1193-8.
41. Richter MJ, Pader P, Gall H, Reichenberger F, Seeger W, Mayer E, Guth S, Kramm T, Grimminger F, Ghofrani HA, Voswinckel R. The prognostic relevance of oxygen uptake in inoperable chronic thromboembolic pulmonary hypertension. Clin Respir J. 2017;11:682-90.

42. Iwase T, Nagaya N, Ando M, Satoh T, Sakamaki F, Kyotani S, Takaki H, Goto $Y$, Ohkita $Y$, Uematsu $M$, et al. Acute and chronic effects of surgical thromboendarterectomy on exercise capacity and ventilatory efficiency in patients with chronic thromboembolic pulmonary hypertension. Heart. 2001;86:188-92.

\section{Publisher's Note}

Springer Nature remains neutral with regard to jurisdictional claims in published maps and institutional affiliations.
Ready to submit your research? Choose BMC and benefit from:

- fast, convenient online submission

- thorough peer review by experienced researchers in your field

- rapid publication on acceptance

- support for research data, including large and complex data types

- gold Open Access which fosters wider collaboration and increased citations

- maximum visibility for your research: over $100 \mathrm{M}$ website views per year

At BMC, research is always in progress.

Learn more biomedcentral.com/submissions 